\title{
Correlation of specific gravity and osmolality of urine in neonates and adults
}

\author{
S LEECH AND M D PENNEY \\ Royal Gwent Hospital, Newport, Gwent, Wales
}

SUMmaRY There is good correlation between the specific gravity and osmolality of urine obtained from neonates and adults, if specimens contain neither protein nor glucose. The regression equations between specific gravity and osmolality, however, differ considerably between the two age groups, maturation to the adult regression occurring within the first 5 years of life. Specifically derived regression equations should therefore be used when predicting urine osmolality from measurement of specific gravity in neonates.

The osmolality of urine is the definitive measure of its concentration. Under normal physiological conditions low urine osmolality equates with hydration, and high urine osmolality with dehydration. The ability of neonates to concentrate urine is lower than that of adults, but the capacity to dilute urine is similar. In a non-uraemic, normonatraemic, and normoglycaemic neonate, therefore, urine osmolality maintained between $75 \mathrm{mosmol} / \mathrm{kg}-300 \mathrm{mosmol} /$ $\mathrm{kg}$ indicates adequate hydration; values outside these ranges suggest overhydration and dehydration, respectively. ${ }^{1}$

The necessity for monitoring urine osmolality in neonates to estimate fluid balance may make heavy demands on the laboratory. Osmometers are expensive and unsuitable for use in the wards without the help of a technician. The specific gravity of urine, traditionally measured in the ward, also reflects concentration, but the prediction of urine osmolality from specific gravity measurement may be unreliable in unskilled hands. ${ }^{2}$ In addition, abnormal concentrations of glucose and protein in the urine cause appreciable increases in specific gravity without affecting osmolality. Finally, measurement by traditional flotation hydrometry requires a large volume of urine. All these factors militate against the use of specific gravity as a measure of urine concentration in neonates.

Despite recent interest in the measurement of specific gravity as an indicator of urine concentration in children, the influence of age on the relation between specific gravity and osmolality has not been defined. ${ }^{3}$ We studied the association between urine specific gravity measured by refractometry, and osmolality, to assess the practicability of measuring the urine concentration of neonates accurately in the ward; we also assessed the influence of age on the relation between specific gravity and osmolality.

\section{Material and methods}

We collected 100 urine samples from 21 neonates in the special care baby unit; the babies had been born at 28 to 32 weeks' gestation and mean (SD) weight was $2100(940) \mathrm{g}$. Single specimens of urine were collected from 22 healthy babies born at full term, 88 children from a paediatric ward (age range 1-70 months), and from 59 healthy adults. The specimens were not taken at a particular time, nor were they preserved. Urine was tested for the presence of glucose and protein using the Ames Uristix, and those specimens showing positive results were excluded.

Osmolality was measured on fresh urine samples by freezing point depression osmometry (Advanced Instruments Model 3D), and specific gravity by a hand held refractometer (Atago, Uricon), which was corrected for temperature before use.

Data were analysed by the method of least squares and by the linear regression method of Deming. ${ }^{+}$Statistical comparison was by Student's $t$ test.

\section{Results}

Fig. 1 shows the $95 \%$ confidence limits of the Deming regression of osmolality to specific gravity for neonates and for adults.

The Deming regression equations were: (i) neon- 
ates $(n=96)$ - osmolality $=20004$ (specific gravity)20025, $r=0.933$, SE Deming regression in Y direction $=28.9$; and (ii) adults $(n=59)$-osmolality = 33194 (specific gravity)-33247, r=0.990, SE Deming regression in $\mathrm{Y}$ direction $=45 \cdot 6$. The Standard Errors of the slopes were 795 and 620 , respectively, and the slopes were significantly different $(\mathrm{p}<0.0001)$.

Fig. 1 shows the results from the samples of urine taken from the neonates born at term, and from the infants aged between 1 and 70 months. Those from the neonates fell within the limits previously defined for the neonates on the special care baby unit, but the infants' data show a progression in the results from the neonatal to the adult association.

To study this progression we ranked the data on specific gravity and osmolality from all infants in 10 month age bands. Using equation 2 , the osmolality of each urine sample was calculated from the measurement of specific gravity. The measured osmolality was expressed as a percentage of the calculated value $(\triangle)$ after correction of values to account for the common intercept of both regression equations which is not at zero, but at an osmolality of $27.5 \mathrm{mosmol} / \mathrm{kg}$.

$$
\Delta=\frac{\text { (measured osmolality }-27 \cdot 5)}{\text { (calculated osmolality }-27 \cdot 5)} \times 100
$$

Fig. 2 shows the association between the mean $\triangle$ (SE) of each age band and the mean age of each band. There is rapid progression towards the adult association of specific gravity and osmolality during the first two years of life which is completed around the fifth year.

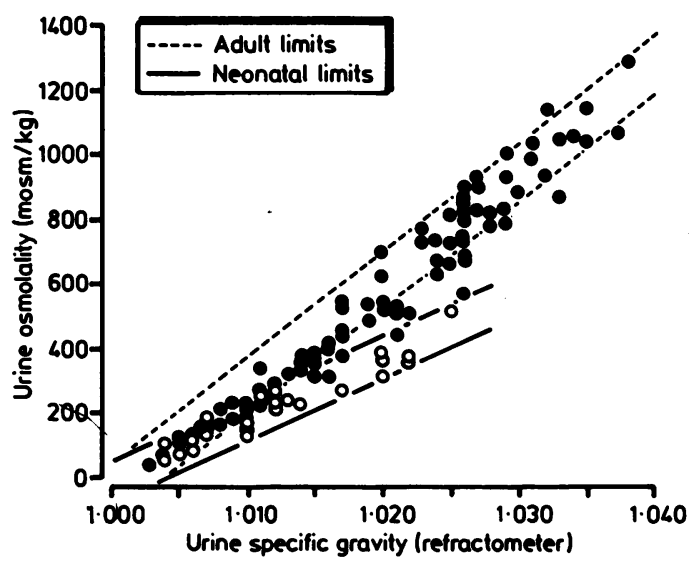

Fig. 1 Association of specific gravity and osmolality of urine in full term healthy neonates $(0)$ and children aged between 1 and 70 months (O); 95\% confidence limits derived from data from neonates in special care baby unit, and healthy adults.

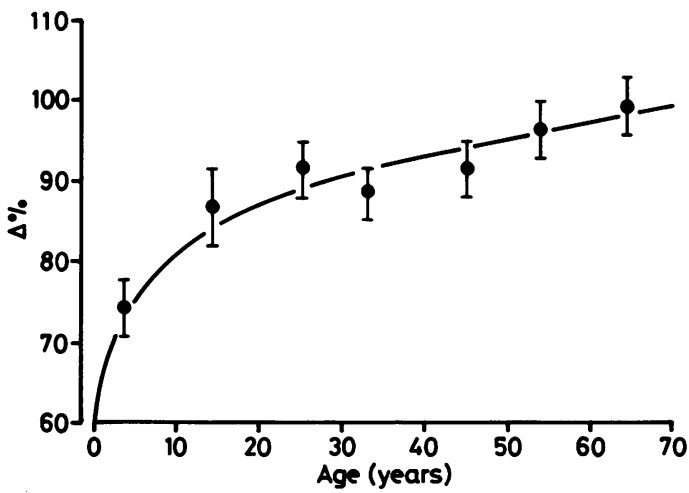

Fig. 2 Change in association of specific gravity and osmolality of urine from neonatal to adult pattern with age.

\section{Discussion}

There is good correlation between urine specific gravity, measured by refractometry, and osmolality in neonates, but the association is different from that found in adults. The change is probably a reflection of the reduced reabsorption of small molecules and low molecular weight proteins by the renal tubules that occur during early infancy and childhood. In particular, the reduced percentage reabsorption of amino acids, ${ }^{5}$ and low molecular weight proteins such as microglobulin, ${ }^{6}$ results in a relatively large increase of specific gravity when compared with osmolality. As proximal tubular function matures, so the association of urine specific gravity and osmolality approaches the adult pattern, which approximates to that produced by the predominant molar constituents of urine-sodium, potassium, and urea. Although the mean age of the infants in the special care baby unit ranged from 28 weeks to term (a period of tubular maturation), the scatter of the values for osmolality and specific gravity of urine suggests that changes in the composition of the urine which were important enough to affect the association between them, do not occur during this period.

As an easy measurement of the specific gravity of urine in the ward refractometry is accurate enough to predict the osmolality of urine in neonates if samples containing protein and glucose are excluded. A specifically derived neonatal regression, however, should be used.

We thank the departments of paediatrics and medical illustration for their help.

\footnotetext{
References

1 Roy RN, Sinclair JC. Hydration of the low birth-weight infant. Clin Perinatol 1975;2:393-417.
} 
2 Galambos JT. Herndon EG Jr. Reynolds GH. Specific gravity determination. Fact or fancy? N Engl J Med 1964:270:506-8.

${ }^{3}$ McCrossin T, Roy LP. Comparison of hydrometry, refractometry, osmometry and Ames N-Multistix SG in estimation of urinary concentration. Aust Paediatr J 1985:21:185-8.

4 Cornblect PJ. Gochman N. Incorrect least-squares regression coefficients in method comparison analysis. Clin Chem 1979:25:432-8.

${ }^{5}$ Brodehl J. Gellisen K. Endogenous renal transport of free amino acids in infancy and childhood. Pediatrics 1968;42: 395-404.

${ }^{6}$ Karlsson FA. Hellsing K. Urinary protein excretion in early infancy. $J$ Paediatr 1976;89:89-90.

Correspondence to Dr M D Penney, Royal Gwent Hospital, Newport, Gwent NPT 2UB, Wales.

Received 16 February 1987

\section{Twenty five years ago}

\section{The measurement and meaning of pyuria}

\section{J M Stansfield (Durham)—Arch Dis Child 1962;37:257-62}

Twenty five years ago the usual method of estimating the degree of pyuria was to place a drop of urine under a microscope and to count the number of cells within a high power field (HPF). While this technique could reveal gross degrees of pyuria, there may be only a few pus cells in some cases of pyelonephritis. In this paper the author pointed to the incongruity of accurately counting cells in blood and cerebrospinal fluid while accepting crude estimations of the cell numbers in urine. He advocated the measurement of pyuria using a counting chamber (Neubauer). In uncentrifuged urine one cell per HPF was found to be equivalent to 250-500 cells per $\mathrm{mm}^{3}$. Cell counts per HPF had higher coefficients of variation than those in which a counting chamber was used. Urine cell counts were made on 2000 consecutive admissions to a general paediatric unit; nearly $5 \%$ of the boys and $27 \%$ of the girls were found to have more than 10 white cells per $\mathrm{mm}^{3}$. These findings reflected the frequency with which naturally voided urines could be contaminated. The importance of pyuria could only be judged from cell counts on clean midstream or catheter specimens. The author considered that a finding of 10 or more cells per $\mathrm{mm}^{3}$ was pathological. By combining cell and bacteriological counts he classified pyuria into three categories: (a) Contamination: whatever the cell count in an ordinary specimen a clean specimen will contain less than 10 cells per $\mathrm{mm}^{3}$ and the culture will be sterile or the bacterial count less than 10000 per $\mathrm{ml}$; (b) Urinary tract infection: a clean specimen contains 10 or more cells per $\mathrm{mm}^{3}$, often in clumps or casts, and the bacterial count will be over 100000 per ml; and (c) Unknown: cells but not organisms are occasionally present in abnormal numbers in clean specimens. The author also recognised that pyuria may sometimes be absent in the presence of urinary tract infection, especially if it has been of long duration, but he considered that occasional difficulties of interpretation did not outweigh the value of the measurements.

Comment. The value of white cell counts in urine is now generally recognised, although demonstraton of an appreciable bacteriuria by culture is essential for a definitive diagnosis. In the acutely ill child the finding on microscopy of a raised urine cell count and visible bacteria may justify immediate treatment while awaiting the results of urine culture. Most laboratories now prefer the Fuchs-Rosenthal counting chamber to the Neubauer. It is, however, to be regretted that so few young doctors of today are encouraged to become self proficient in the simple techniques that used to be employed in ward side rooms. 